\title{
hsa-miR-429 targets CBX8 to promote cell apoptosis in diffuse large B-cell lymphoma
}

\author{
YAN LIANG ${ }^{1,2^{*}}$, ZHUO-JUN YU ${ }^{1,2^{*}}$, MIN LIU ${ }^{1,2}$, HUI-MIN LIU ${ }^{1,2}$, JIANG-ZHAO ZHANG $^{1,2}$, \\ TAO XIONG $^{1,2}$, YUAN-YAN TANG ${ }^{1,2}$ and ZHI-PING HUANG ${ }^{1,2}$ \\ ${ }^{1}$ Department of Hematology, Jingzhou Central Hospital, Institute of Hematology; \\ ${ }^{2}$ The Second Clinical Medical College, Yangtze University, Jingzhou, Hubei 434020, P.R. China
}

Received May 13, 2021; Accepted September 14, 2021

DOI: $10.3892 / \mathrm{mmr} .2021 .12497$

\begin{abstract}
Diffuse large B-cell lymphoma (DLBCL) is the most common type of non-Hodgkin lymphoma worldwide. Several studies have indicated that Homo sapiens (hsa)-microRNA (miR)-429 exerts a tumor-suppressive effect on a variety of malignant tumors. To the best of our knowledge, the molecular function and mechanism of action of hsa-miR-429 in DLBCL have not been evaluated to date. The present study demonstrated that the expression of hsa-miR-429 in DLBCL cells was significantly reduced. hsa-miR-429 inhibited the proliferation of the DLBCL cell lines, SUDHL-4 and DB, and promoted apoptosis. A dual luciferase reporter assay was used to demonstrate that chromobox 8 (CBX8) was the target gene of hsa-miR-429. Overexpression of CBX8 promoted the proliferation of SUDHL- 4 and DB cells and inhibited apoptosis, thereby playing a cancer-promoting role. Transfection of hsa-miR-429 mimic into DB cells overexpressing CBX8 antagonized the effect of CBX8 on the proliferation of DB cells. Moreover, the apoptotic rate was increased in DB cells overexpressing CBX8 and transfected with hsa-miR-429 mimic, while the proportion of cells in the $\mathrm{G}_{2} / \mathrm{M}$ phase was significantly reduced. These results demonstrated the antagonistic effect of hsa-miR-429 on the oncogenic function of CBX8. Therefore, in DLBCL, the tumor suppressor effect of hsa-miR-429 may be achieved by targeted downregulation of CBX8, suggesting that hsa-miR-429 may be used as a diagnostic marker and a potential nucleic acid drug for DLBCL. CBX8 may also represent an effective therapeutic target for DLBCL.
\end{abstract}

Correspondence to: Dr Zhi-Ping Huang, Department of Hematology, Jingzhou Central Hospital, Institute of Hematology, Yangtze University, 1 Renmin Road, Jingzhou, Hubei 434020, P.R. China

E-mail: 191060635@qq.com

${ }^{*}$ Contributed equally

Key words: hsa-microRNA-429, chromobox 8, diffuse large B-cell lymphoma, apoptosis, cell proliferation

\section{Introduction}

Diffuse large B-cell lymphoma (DLBCL) is the most common type of non-Hodgkin lymphoma globally, accounting for $30-40 \%$ of all cases in different geographic regions. DLBCL is an aggressive disease with heterogeneous genes, phenotypes and clinical characteristics (1). The patients most often present with a rapidly growing tumor mass in a single or multiple lymph nodes, and even outside the lymph nodes. Combining rituximab and cyclophosphamide, Adriamycin, vincristine and prednisone (CHOP regimen) can significantly improve the prognosis of patients with DLBCL, and the 3-year recurrence-free survival period can reach $65-80 \%$. However, $30-40 \%$ of patients with DLBCL will relapse or develop refractory disease, which is still the main cause of patient-related deaths. Therefore, molecular discovery may be the best way forward for designing new therapies in precision medicine. In addition to traditional diagnostic functions, it is also necessary to explore prognostic markers and potential therapeutic targets (2).

MicroRNA (miRNA/miR) is a type of endogenous non-coding RNA, 18-25 nucleotides in length, which is ubiquitously expressed both intra- and extracellularly. Its biological function can lead to mRNA degradation or translation inhibition, thereby playing an important regulatory role in animal and plant cells (3). This post-transcriptional regulation can directly affect the function of target genes and participate in the physiological and pathological processes of multiple systems of the body, including the digestive system. At present, a large number of clinical and basic studies have revealed that miRNA can act as an oncogene or tumor suppressor and has key functions in tumorigenesis and malignant progression $(4,5)$.

Several studies have suggested that Homo sapiens (hsa)-miR-429 acts as a tumor suppressor in a variety of malignant tumors. hsa-miR-429 can inhibit the Raf/MEK/ERK pathway by targeting CRK like proto-oncogene adaptor protein to reduce the migration of liver cancer cells and reverse epithelial-mesenchymal transition (6,7). Moreover, it can inhibit hepatocyte proliferation and liver regeneration by targeting the JUN/MYC/BCL2/cyclin D1 axis (8). hsa-miR-429 exerts a tumor-suppressive effect by targeting fascin actin-bundling protein 1 in gastric cancer cells (9). 
In addition, the expression of hsa-miR-429 in patients with bladder cancer surviving for 5 years was revealed to be significantly higher compared with that in deceased patients, and it was a potential prognostic indicator of bladder cancer (10). hsa-miR-429 can also promote the proliferation of bladder cancer cells by inhibiting cyclin-dependent kinase inhibitor 2B (11). hsa-miR-429 can inhibit the progression and metastasis of osteosarcoma by targeting zinc finger E-box binding homeobox 1 (12). hsa-miR-429 also regulates the proliferation and apoptosis of Wilms' tumor cells by targeting c-Myc, reduces the perineural invasion of pancreatic cancer by inhibiting neurotrophic factor-3, and inhibits the migration and invasion of esophageal squamous cell carcinoma cells by targeting Slug (13-15). Previous studies have suggested that long non-coding RNA (lncRNA) small nucleolar RNA host gene 6 (SNHG6) is a regulatory factor of hsa-miR-429. When the expression levels of SNHG6 increase, the concentration of free hsa-miR-429 in the cell is significantly reduced, which promotes the progression of Wilms' tumor (16).

The role of hsa-miR-429 in breast cancer is controversial. A previous study has revealed that hsa-miR-429 inhibits the proliferation and invasion of breast cancer cells by inhibiting the Wnt/ $\beta$-catenin signaling pathway (17). Moreover, in erb-b2 receptor tyrosine kinase 2 positive $\left(\mathrm{HER}^{2+}\right)$ breast cancer, hsa-miR-429 could regulate the hypoxia-inducible factor 1 subunit $\alpha(\mathrm{HIF} 1 \alpha)$ pathway by directly targeting von Hippel-Lindau tumor suppressor mRNA, an important molecule that degrades HIF1 $\alpha$. Overexpression of hsa-miR-429 in $\mathrm{HER}^{2+}$ breast cancer was revealed to lead to an increase in breast cancer cell proliferation and migration, whereas silencing of hsa-miR-429 could delay tumor growth. This suggests that hsa-miR-429 may play a dual role in different tumors (18). However, to the best of our knowledge, the molecular function and mechanism of action of hsa-miR-429 in DLBCL have never been evaluated.

\section{Materials and methods}

Cell culture. The DLBCL cell lines, SUDHL-4 and DB, were provided by The Chinese Academy of Sciences Stem Cell Bank. Both are suspension cells, cultured with $90 \%$ RPMI-1640 + 10\% FBS (both from HyClone; Cytiva) at $37^{\circ} \mathrm{C}$ and $5 \% \mathrm{CO}_{2}$.

Clinical sample collection. A total of 10 newly diagnosed patients with DLBCL admitted to the Department of Hematology, Jingzhou Central Hospital from January 2019 to June 2019 were included in the present study. There were 7 males and 3 females with a median age of 65 years (50-76 years). Inclusion criteria were as follows: lymph node enlargement was the first manifestation of all patients. The lymph nodes with suspected lesions were completely resected and further analyzed by immunohistochemistry after paraffin embedding. The immunohistochemical analysis included CD20, CD3, CD5, CD10, CD45, CD30, Bcl-2, Bcl-6, Ki-67, Mum-1, and MYC. The pathological diagnosis was in line with the World Health Organization (WHO) diagnostic criteria for hematopoietic and lymphoid tissue tumors in 2008. Exclusion criteria were as follows: previous hematopoietic and lymphoid tumor. Normal tissue was obtained from patients hospitalized at the Department of Hematology at the same period, with lymphadenopathy as the main manifestation, and lymphadenitis confirmed by immunohistochemical analysis after complete excision of disaffected lymph nodes. The samples used in this study were collected from the discarded samples after lymph node biopsy of patients without affecting the disease diagnosis and treatment of patients, with the consent of patients and their family members. Ethical approval was obtained from The Ethics Committee of Jingzhou Central Hospital (Jingzhou, China). Written informed consent was obtained from the patients for their anonymized information to be published in this article. All patients consented to participate in the study by having their samples used in clinical research.

Quantitative PCR ( $q P C R)$. Total RNA was extracted using TRIzol ${ }^{\circledR}$ reagent (Thermo Fisher Scientific, Inc.) from DLBCL tissue. Bulge-Loop hsa-miR-429 Primer Set (cat. no. MQPS0001297-1-100) was used to quantify hsa-miR-429 by qPCR with SYBR-Green (FP411-02; TIANGEN). The thermocycling conditions were as follows: Initial denaturation, $95^{\circ} \mathrm{C}, 15 \mathrm{~min}$; followed by a 45 -cycle, $94^{\circ} \mathrm{C}, 20 \mathrm{sec}, 60^{\circ} \mathrm{C}, 34 \mathrm{sec}$ ). The primer sequences of U6 (internal reference) were as follows: forward, 5'-CTCGCTTCGGCAGCACA-3' and reverse, 5'-AACGCTTCACGAATTTGCGT-3'. The aforementioned kit was purchased from Guangzhou RiboBio Co., Ltd. It was used according to the manufacturer's instructions. The relative expression levels were calculated using the $2^{-\Delta \Delta \mathrm{Cq}}$ method (19).

Transfection. hsa-miR-429 mimic (cat. no. miR10001536-1-5; sequence: 5'-UAAUACUGUCUGGUAAAACCGU-3'), mimic negative control (cat. no. miR1N0000001-1-5; sequence: 5'-UUUGUACUACACAAAAGUACUG-3'), hsa-miR-429 inhibitor (cat. no. miR20001536-1-5; sequence: 5'-ACGGUU UUACCAGACAGUAUUA-3') and inhibitor negative control (cat. no. miR2N0000001-1-5; sequence: 5'-CAGUACUUU UGUGUAGUACAAA-3') were purchased from Guangzhou RiboBio Co., Ltd. miRNA mimic and inhibitor were transfected into cell culture plates with Ribofect ${ }^{\mathrm{TM}}$ CP Reagent (cat. no. C10511-05; Guangzhou RiboBio Co., Ltd.), and cultured at $37^{\circ} \mathrm{C}$ for $48 \mathrm{~h}$. The transfection concentrations were $50 \mathrm{nM}$ (miRNA mimic) and $100 \mathrm{nM}$ (miRNA inhibitor). A total of $20 \mu \mathrm{M}$ miRNA mimic or inhibitor was diluted with $1 \mathrm{X}$ Ribofect ${ }^{\mathrm{TM}}$ CP Buffer as recommended and gently mixed. The Ribofect ${ }^{\mathrm{TM}}$ CP Reagent was added, mixed gently, and incubated at room temperature for 10-15 min. The Ribofect ${ }^{\mathrm{TM}} \mathrm{CP}$ mixture was added to the complete medium and mixed gently. Subsequently, the culture plate was placed in a $\mathrm{CO}_{2}$ incubator at $37^{\circ} \mathrm{C}$ for $48 \mathrm{~h}$, and the related cellular and molecular experiments were carried out.

Cell proliferation experiment. Cell proliferation was evaluated using a Cell Counting Kit-8 (CCK-8; cat. no. C0037; Beyotime Institute of Biotechnology) assay. A total of $1 \times 10^{3}$ cells in $100 \mu \mathrm{l}$ medium were plated into a 96-well plate. After $48 \mathrm{~h}$ incubation at $37^{\circ} \mathrm{C}, 10 \mu \mathrm{l}$ of CCK8 was added to each well and incubated at $37^{\circ} \mathrm{C}$ in dark for $1 \mathrm{~h}$. The absorbance was measured at $450 \mathrm{~nm}$.

Cell colony formation assay. The cells were seeded into a 6 -well plate at a concentration of $1 \times 10^{3}$ cells in $2 \mathrm{ml}$ complete 
medium and incubated at $37^{\circ} \mathrm{C}, 5 \% \mathrm{CO}_{2}$ for 14 days. The medium was changed every 3 days. After the formation of visible colonies, the cells were fixed with $4 \%$ formaldehyde for $20 \mathrm{~min}$ at room temperature and stained with $0.1 \%$ crystal violet for 10-15 min at room temperature. After washing away the background color, photomicrographs were obtained and the number of colonies was counted by eye.

Apoptosis analysis. The cells were stained with Annexin V-FITC Apoptosis Detection kit (cat. no. C1062L; Beyotime Institute of Biotechnology) at room temperature in dark for $10 \mathrm{~min}$. The kit was used according to the manufacturer's instructions. The apoptotic rate was detected by flow cytometry (BD LSRFortessa and FlowJo7.6.5; BD Biosciences).

Cell cycle analysis. The cells at $70 \%$ confluent were collected by centrifugation at $1,000 \mathrm{x}$ for $5 \mathrm{~min}$ at $22^{\circ} \mathrm{C}$ and fixed with ice-cold $70 \%$ ethanol at $4^{\circ} \mathrm{C}$ for $30 \mathrm{~min}$ or longer. The cells were stained with Cell Cycle and Apoptosis Analysis kit (cat. no. C1052; Beyotime Institute of Biotechnology) at $37^{\circ} \mathrm{C}$ in dark for $30 \mathrm{~min}$. The kit was used according to the manufacturer's instructions. The cell cycle distribution was detected by flow cytometry (BD LSRFortessa and FlowJo7.6.5; BD Biosciences).

Target gene prediction. TargetScan Human 7.2 (version 7.2; targetscan.org/vert_72/) was used to predict the target gene of hsa-miR-429.

Dual luciferase reporter gene assay. hsa-miR-429 mimic (cat. no. miR10001536-1-5; Sequence: 5'-UAAUACUGUCUGGU AAAACCGU-3'), mimic negative control (cat. no.miR1N0000001-1-5; sequence:5'-UUUGUACUACACAAA AGUACUG-3'), hsa-miR-429 inhibitor (cat . no. miR20001536-1-5; sequence: 5'-ACGGUUUUACCAGAC AGUAUUA-3') and inhibitor negative control (cat. no. miR2N0000001-1-5; sequence: 5'-CAGUACUUUUGUG UAGUACAAA-3') were purchased from Guangzhou RiboBio Co., Ltd. A dual luciferase reporter assay was used to verify the binding of hsa-miR-429 to the 3 ' untranslated region (UTR) of CBX8. The 3'-UTR wild-type (WT) sequence and 3'-UTR mutant (MUT) sequence of CBX8 were cloned into the pGL6-miR plasmid (cat. no. D2106; Beyotime Institute of Biotechnology). The recombinant plasmid $(2 \mu \mathrm{g})$ and hsa-miR-429 mimic (50 $\mathrm{nM}$ ) or mimic negative control (50 nM) were co-transfected into $293 \mathrm{~T}$ cells at $40 \%$ confluent (supplied by the Chinese Academy of Sciences Stem Cell Bank) seeded in a 6-well plate. The transfection reagent was Lipofectamine 3000 (cat. no. L3000008; Thermo Fisher Scientific). After $48 \mathrm{~h}$ at $37{ }^{\circ} \mathrm{C}$, the relative luciferase activity was detected with Dual-Luciferase Reporter Assay Systems (Promega Corporation). The results were normalized with Renilla luciferase activity.

Overexpression and knockdown of $C B X 8$. The coding sequence of the human CBX8 (gene ID, 57332) was cloned into the pcDNA3.1 (Invitrogen; Thermo Fisher Scientific, Inc.) vector to generate an overexpression vector. Small interfering RNA (siRNA) was used to knock down the expression of the CBX8 gene. CBX8 siRNA was purchased from Guangzhou

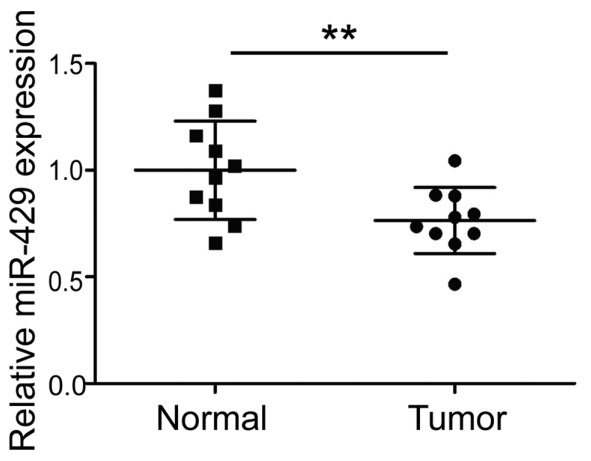

Figure 1. Expression of hsa-miR-429 in DLBCL cells is significantly reduced. The expression of hsa-miR-429 in tumor and normal tissue samples from patients with DLBCL was detected by reverse transcription-quantitative PCR. ${ }^{* *} \mathrm{P}<0.01$. DLBCL, diffuse large B-cell lymphoma; hsa, Homo sapiens; miR, microRNA.

RiboBio Co., Ltd. In order to avoid off-target effects, two different pairs of CBX8 siRNA were selected for the experiment (cat. no. siG000057332A-1-5 and siG000057332B-1-5; siG000057332A-1-5 target sequence: 5'-GAGGAAAACATC CTGGATGC-3'; and siG000057332B-1-5 target sequence: 5'-TGCTCGCAGCCTTTGAGGAA-3'). siN0000002-1-5 (Guangzhou RiboBio Co., Ltd.; target sequence: 5'-TTCTCC GAACGTGTCACGT-3') was used as a negative control. Cells were transfected with the CBX8 expression vector $(6 \mu \mathrm{g}$ DNA per $10-\mathrm{cm}$ dish) and siRNA (100 $\mathrm{nM})$ to overexpress or knock down the expression of CBX8 by PolyFect Transfection Reagent (cat. no. 301108; QIAGEN) at room temperature, respectively. The transfection lasted $6 \mathrm{~h}$. The medium containing the transfection reagent was removed and replaced with fresh medium. After culturing in a $\mathrm{CO}_{2}$ incubator at $37^{\circ} \mathrm{C}$ for $48 \mathrm{~h}$, the subsequent experiments were carried out.

Western blot analysis. The anti-CBX8 (cat. no. 61237), anti-GAPDH (HRP-conjugated; cat. no. HRP-60004) and goat anti-rabbit IgG (HRP-conjugated; cat. no. SA00001-2) antibodies were purchased from ProteinTech Group, Inc. The total protein was extracted by RIPA lysis buffer (P0013B, Beyotime Biotechnology). The protein was quantified by BCA method. A total of $10 \mu \mathrm{g}$ of protein was loaded per lane of $10 \%$ SDS-PAGE gel. PVDF membrane was used. The PVDF membrane was blocked with $5 \%$ skimmed milk at room temperature for $30 \mathrm{~min}$. The primary antibodies were diluted at a ratio of 1:1,000 and the secondary antibodies were diluted at a ratio of 1:5,000. PVDF membrane was incubated with primary and secondary antibodies at room temperature for $1 \mathrm{~h}$ each. It was washed with TBST with $0.05 \%$ Tween-20. Western blotting was performed according to conventional methods. Finally, ECL chemiluminescence solution (SuperSignal ${ }^{\mathrm{TM}}$ West Pico PLUS, 34577; Thermo Fisher Scientific, Inc.) was used to develop the image.

Statistical analysis. All experiments were repeated three times. The data were analyzed using SPSS 17.0 software (SPSS, Inc.) package and are presented as the mean \pm SD. One-way ANOVA was used to analyze the data and S-N-K method was used as the post hoc test. $\mathrm{P}<0.05$ was considered to indicate a statistically significant difference. Graphs were 
A

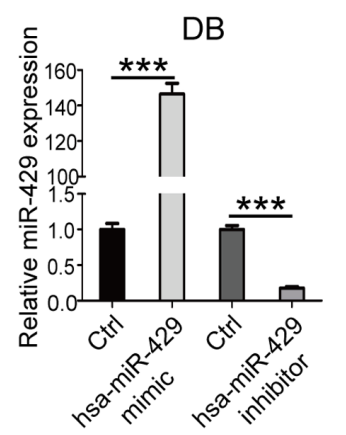

C

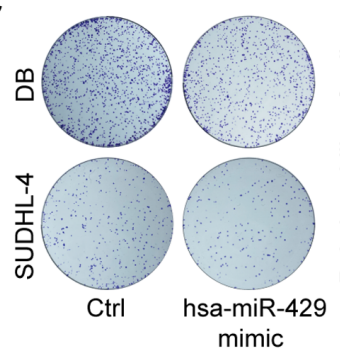

D
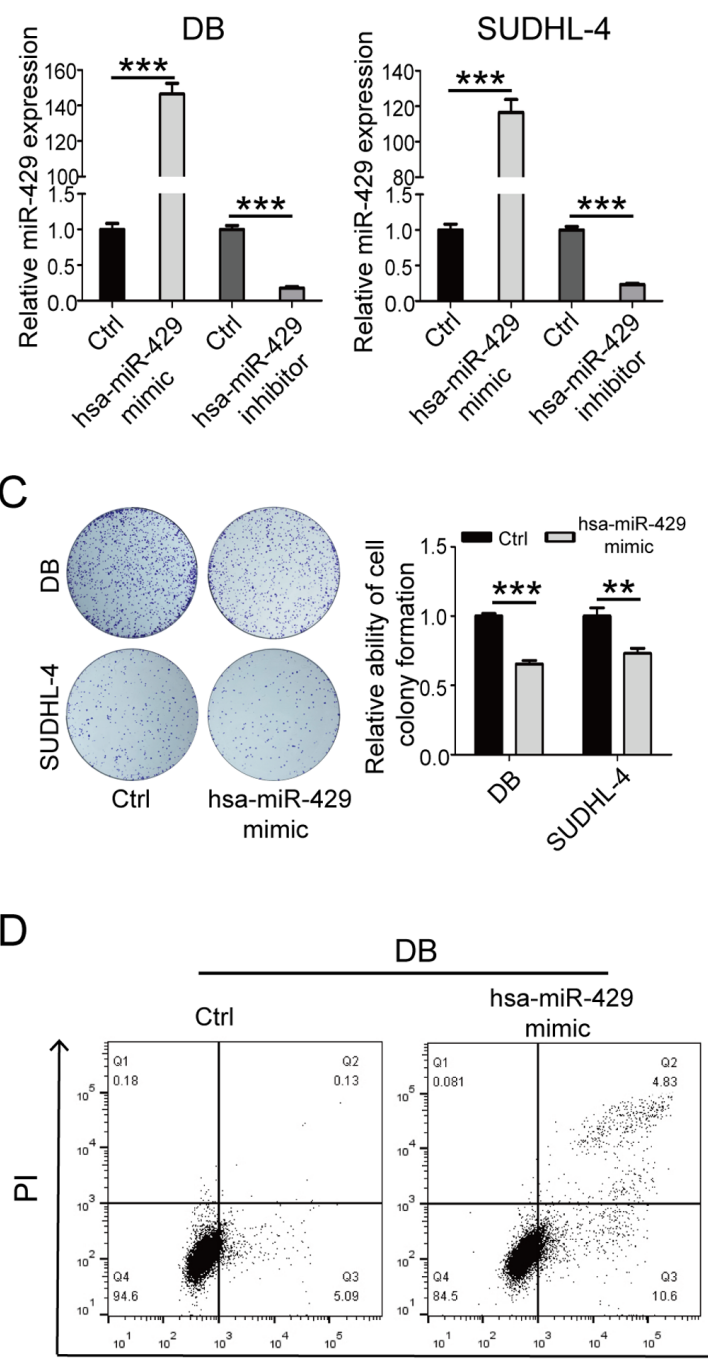

B
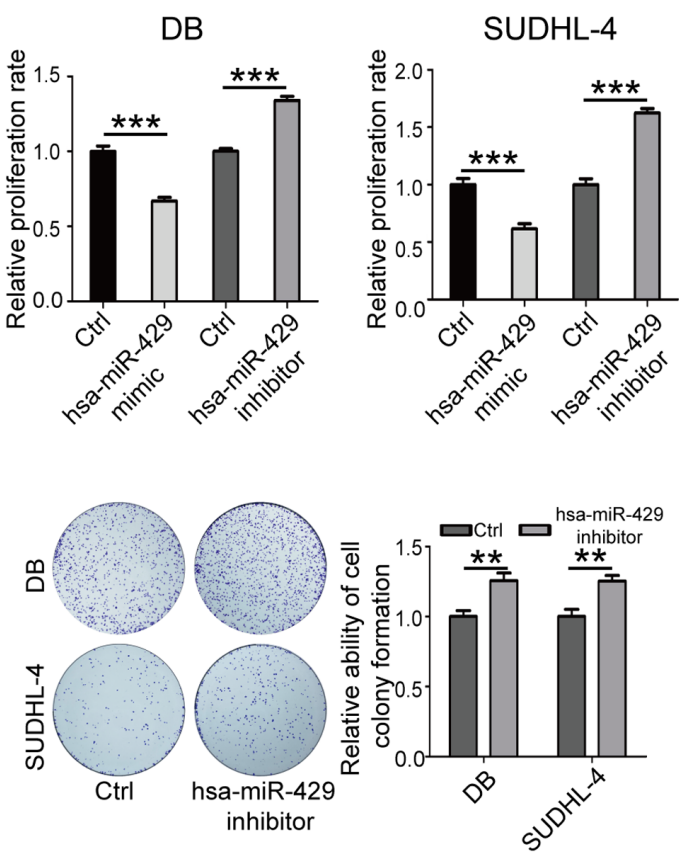

DB

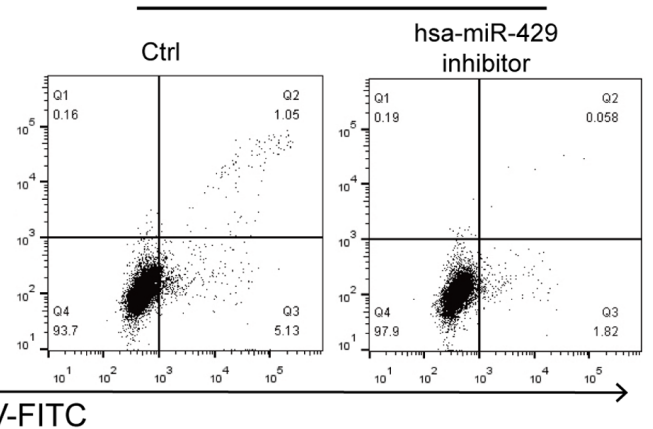

E
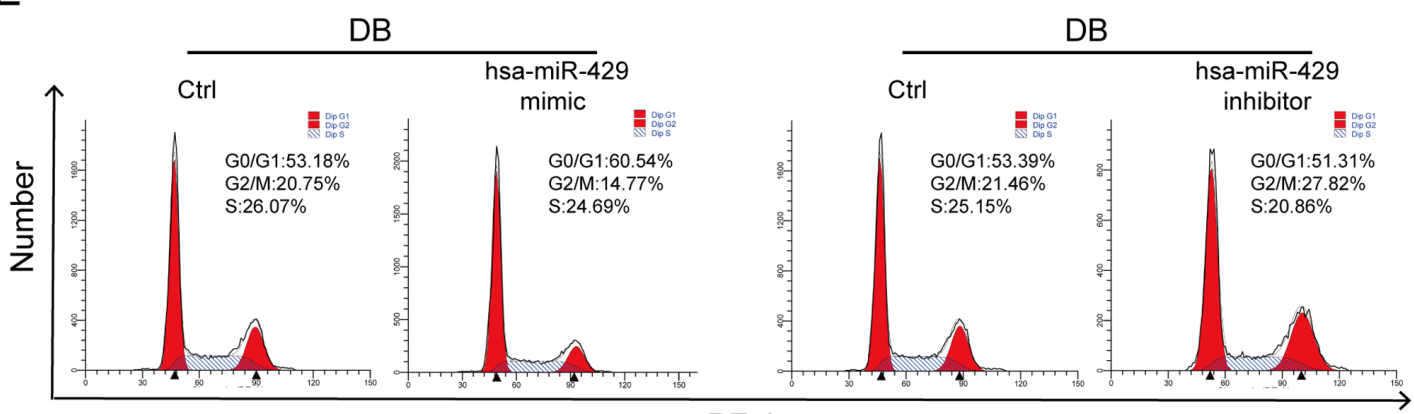

PE-A

Figure 2. hsa-miR-429 inhibits the proliferation of SUDHL-4 and DB cells and promotes apoptosis. (A) The transfection effect was detected by reverse transcription-quantitative PCR. (B) Cell Counting Kit-8 assays were used to detect the effect of hsa-miR-429 on the proliferation of diffuse large B-cell lymphoma cells. (C) Results of cell colony formation assays. (D) The effect of hsa-miR-429 on the cell apoptotic rate was detected by flow cytometry. (E) The effect of hsa-miR-429 on the cell cycle distribution was detected by flow cytometry. ${ }^{* *} \mathrm{P}<0.01$ and ${ }^{* * * *} \mathrm{P}<0.001$. hsa, Homo sapiens; miR, microRNA; Ctrl, control.

generated using GraphPad Prism 5 software (GraphPad Software, Inc).

\section{Results}

Expression of hsa-miR-429 is significantly downregulated in DLBCL tissue. Tumor and normal tissue samples from
10 patients with DLBCL were collected. The expression of hsa-miR-429 was detected by qPCR. The results revealed that hsa-miR-429 expression was significantly reduced in DLBCL tissue ( $\mathrm{P}<0.01$; Fig. 1). tosis of SUDHL-4 and DB cells. SUDHL-4 and DB cells were 
A

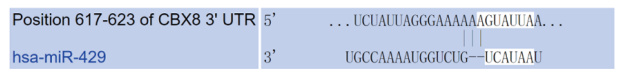

B

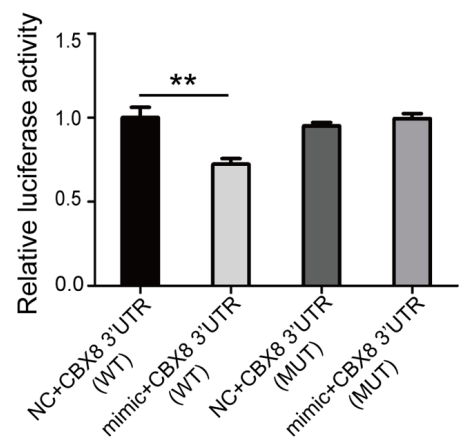

Figure 3. CBX8 is the target gene of hsa-miR-429. (A) TargetScan was used to predict the binding site of hsa-miR-429 in the CBX8 3'-UTR. (B) Dual luciferase reporter gene assay verified the binding site of hsa-miR-429 in the CBX8 3'-UTR. ${ }^{* *} \mathrm{P}<0.01$. hsa, Homo sapiens; miR, microRNA; CBX8, chromobox 8; UTR, untranslated region; WT, wild-type; MUT, mutant.

transfected with hsa-miR-429 mimic and inhibitor (Fig. 2A). CCK-8 and colony formation assay results revealed that transfection with hsa-miR-429 mimic significantly inhibited cell proliferation, while transfection with hsa-miR-429 inhibitor significantly promoted cell proliferation (Fig. 2B and C). Moreover, the detection of the cell apoptotic rate and cell cycle distribution by flow cytometry indicated that transfection with hsa-miR-429 mimic resulted in a significant increase in the cell apoptotic rate and decrease in the proportion of cells in the $\mathrm{G}_{2} / \mathrm{M}$-phase of the cell cycle. The experimental groups transfected with hsa-miR-429 inhibitor revealed the opposite results (Fig. 2D and E). The aforementioned results indicated that hsa-miR-429 could inhibit the proliferation and promote the apoptosis of DLBCL cells.

CBX8 is the target gene of hsa-miR-429. TargetScanHuman 7.2 (http://www.targetscan.org/vert_72/) predicted that CBX8 may be the target gene of hsa-miR-429 (Fig. 3A). CBX8 exerts cancer-promoting effects on a variety of malignant tumors, such as liver, colon and esophageal cancer (20-25). The WT and MUT 3'-UTR (mutated at positions 617-623) sequences of CBX8 were cloned into the pGL6-miR vector. miR-429 mimic and mimic negative control were co-transfected into 293T cells with the pGL6-miR 3'-UTR WT and pGL6-miR 3'-UTR MUT plasmid. Luciferase activity was then detected using a dual luciferase detection system. The results demonstrated that the luciferase activity of the hsa-miR-429 mimic + pGL6-miR 3'-UTR WT group was significantly reduced. After mutating positions 617-623, the luciferase activity was not significantly altered (Fig. 3B). This result indicated that hsa-miR-429 regulated its transcription levels by targeting the 617-623 position of the 3'-UTR of CBX8.

$C B X 8$ promotes the proliferation and inhibits the apoptosis of SUDHL-4 and DB cells. Overexpression of CBX8 in SUDHL-4 and DB cells led to a significant increase in cell proliferation (Fig. 4A-D), a distinct decrease in the cell apoptotic rate (Fig. $4 \mathrm{E}$ ) and a marked increase in the proportion of cells in the $\mathrm{G}_{2} / \mathrm{M}$ phase (Fig. $4 \mathrm{~F}$ ). CBX8 knockdown had the opposite effects. The aforementioned results indicated that CBX8 may promote oncogenesis in DLBCL cells.

hsa-miR-429 antagonizes the effect of CBX8 on DB cell proliferation and inhibition of apoptosis. hsa-miR-429 mimic was transfected into DB cells overexpressing CBX8. Western blot analysis demonstrated that hsa-miR-429 mimic significantly downregulated the protein and mRNA expression levels of CBX8 in DB cells (Fig. 5A and B). The results of the cell proliferation experiments and cell colony formation assays revealed that hsa-miR-429 mimic antagonized the positive effect of CBX8 on the proliferation of DB cells (Fig. 5C and D). In addition, the apoptotic rate of DB cells overexpressing CBX8 transfected with hsa-miR-429 mimic was increased (Fig. 5E), and the proportion of cells in the $\mathrm{G}_{2} / \mathrm{M}$ phase was significantly reduced (Fig. 5F). The aforementioned results demonstrated the antagonistic effect of hsa-miR-429 on the oncogenic role of CBX8.

\section{Discussion}

miRNA is widely expressed in animal and plant cells. miRNA can be combined with lipids or lipoproteins and other components in cells to form microvesicles, which can be disseminated outside of the cell and enter the peripheral blood circulation. It can also enter recipient cells through endocytosis, thereby exerting specific biological effects and regulating target genes $(4,5)$. Previous studies have revealed that different types of malignant tumors may aberrantly express specific tumor-associated miRNA molecules $(4,5)$. The aberrant expression of miRNA can play a key role in multiple biological processes, such as tumor cell survival, invasion and metastasis.

In the present study, hsa-miR-429 played a tumor-suppressive role in DLBCL. High levels of hsa-miR-429 inhibited cell proliferation, promoted apoptosis and changed the distribution of cells in the cell cycle. Bioinformatics prediction revealed that CBX8 was the target of hsa-miR-429. The dual luciferase experiment demonstrated that hsa-miR-429 regulated its transcription levels by targeting the 617-623 positions in the 3'-UTR of CBX8. It has been reported that, in hepatocellular carcinoma, $\mathrm{CBX} 8$ exhibits oncogenic activity through AKT/ $\beta$-catenin activation $(20,21)$. It has also been demonstrated to interact with Y-Box binding protein 1 (YBX1) to promote the proliferation of liver cancer cells (22). CBX8 promoted tumorigenesis and radiation tolerance of esophageal squamous cell carcinoma cells by targeting APAF1 (23). It has also been reported that CBX8 participates in the DNA repair process and promotes the occurrence of esophageal cancer (24). In addition, CBX8 has been revealed to promote the progression and drug resistance of colon cancer, breast cancer, leukemia, bladder cancer and prostate cancer (25-29). To the best of our knowledge, the role of CBX8 in DLBCL has not yet been studied. The present study demonstrated that $\mathrm{CBX} 8$ also played a role in promoting DLBCL. Overexpression of CBX8 in SUDHL-4 and DB cells led to a significant increase in cell proliferation, a notable decrease in the cell apoptotic rate and a marked increase in 
A

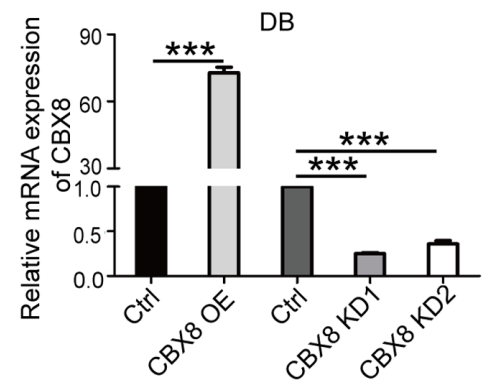

B

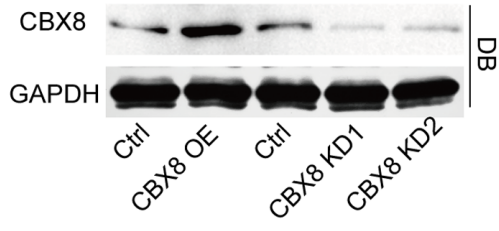

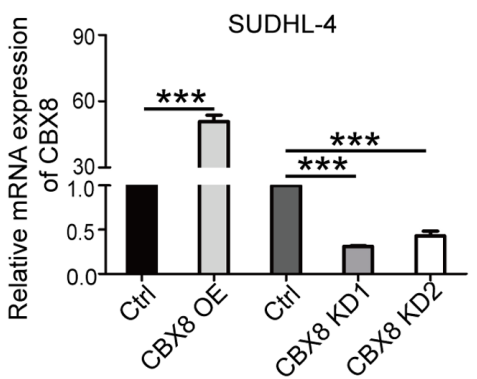

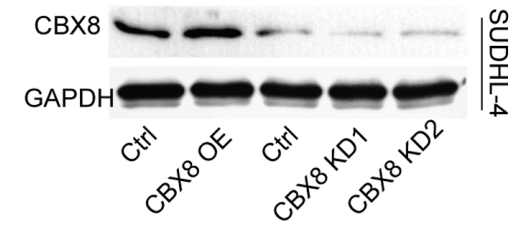

C
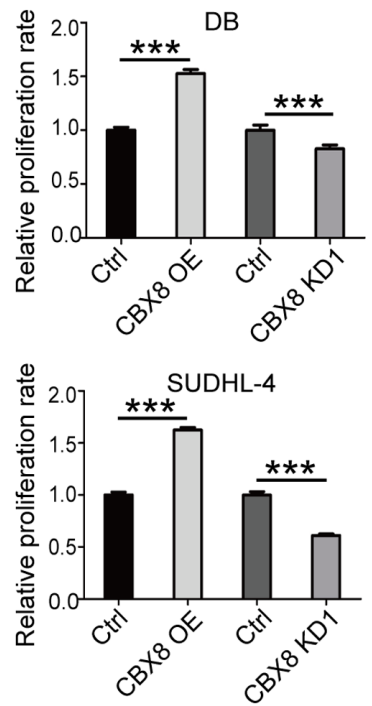

D
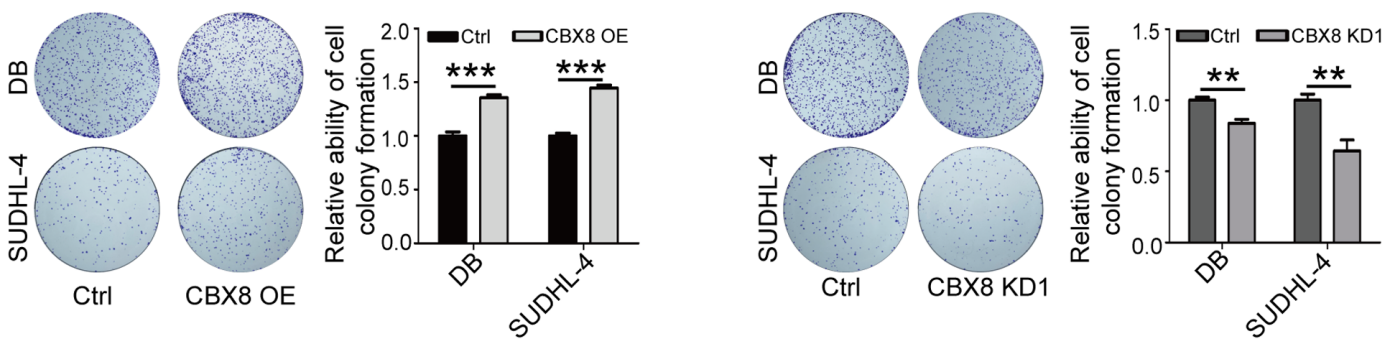

E
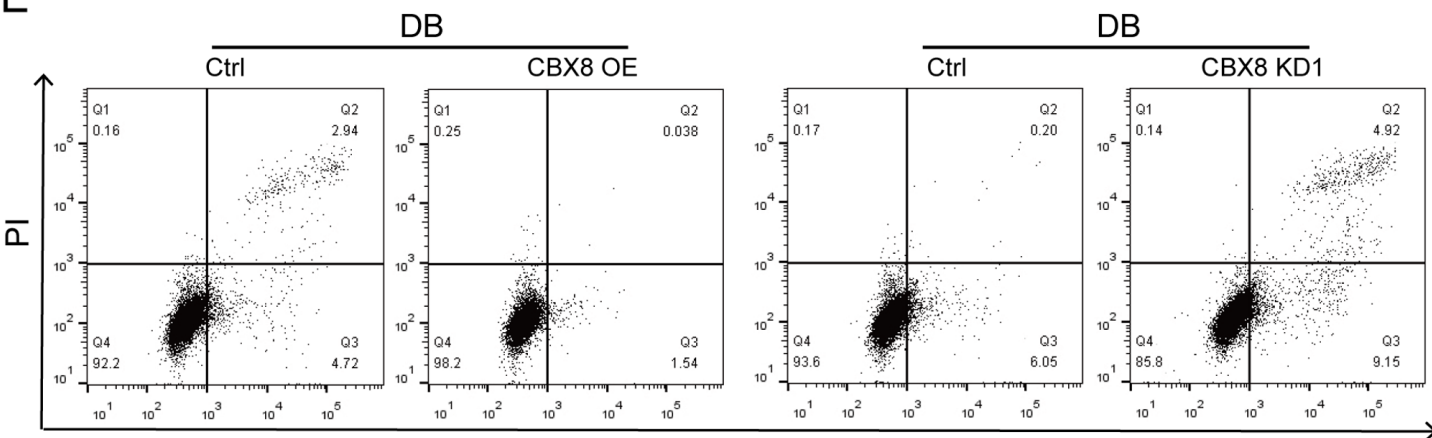

F

Annexin V-FITC
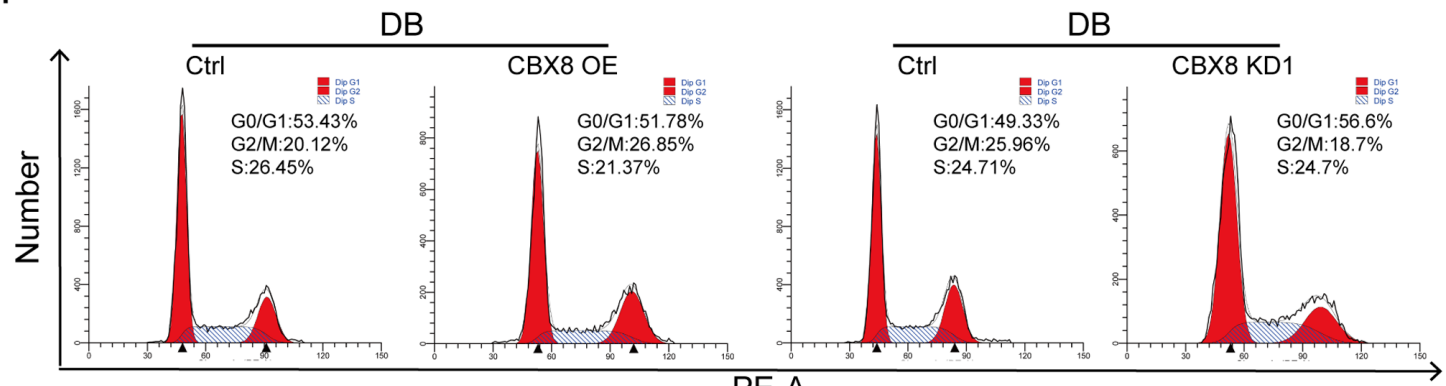

PE-A

Figure 4. CBX8 promotes the proliferation of SUDHL-4 and DB cells and inhibits apoptosis. (A) The transfection effect was detected by reverse transcription-quantitative PCR. (B) Western blot analysis demonstrated that CBX8 overexpression and knockdown were successful. (C) Cell Counting Kit-8 assays were carried out to detect the effect of CBX8 on the proliferation of diffuse large B-cell lymphoma cells. (D) Results of cell colony formation assay. (E) The effect of CBX8 on the apoptotic rate of cells was detected by flow cytometry. (F) The effect of CBX8 on the cell cycle distribution was detected by flow cytometry. ${ }^{* *} \mathrm{P}<0.01$ and ${ }^{* * *} \mathrm{P}<0.001$. CBX8, chromobox 8; OE, overexpression; KD, knockdown; Ctrl, control.

the proportion of cells in the $\mathrm{G}_{2} / \mathrm{M}$ phase of the cell cycle. Transfection of hsa-miR-429 mimic into cells reversed these results. Therefore, in DLBCL, the tumor-suppressive effect of hsa-miR-429 was achieved by targeted downregulation of
CBX8. This result indicated that hsa-miR-429 may be used as a diagnostic marker and a potential nucleic acid drug for DLBCL. CBX8 may also become an effective therapeutic target for DLBCL. 
A

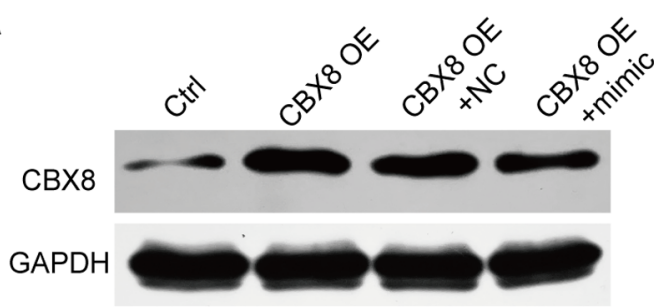

C

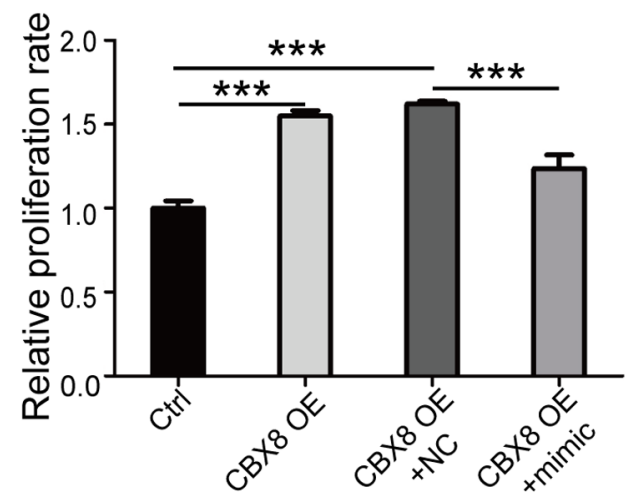

E
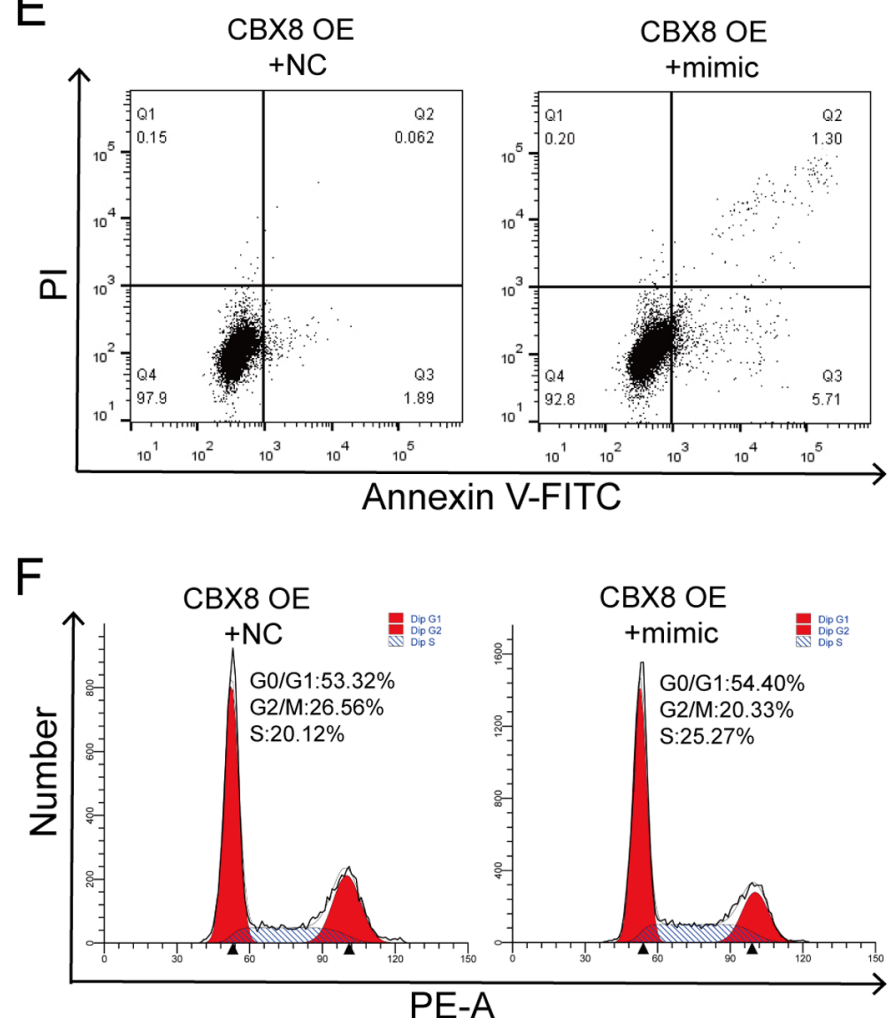

B

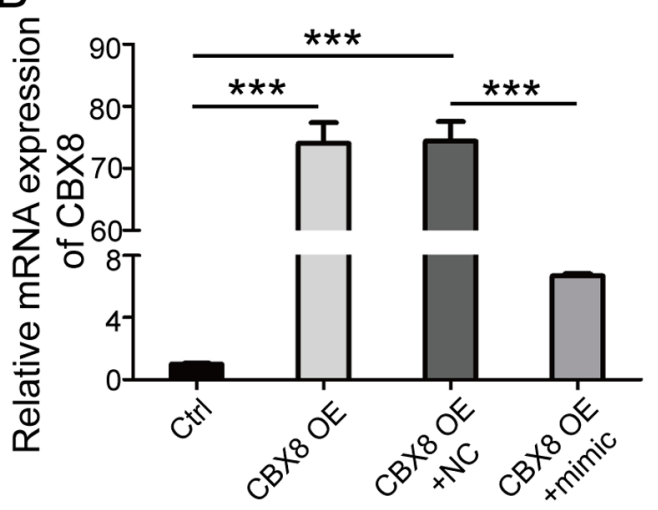

D
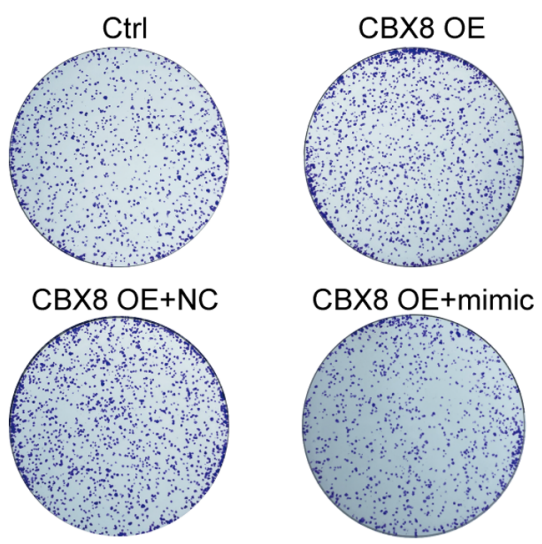

CBX8 OE+mimic

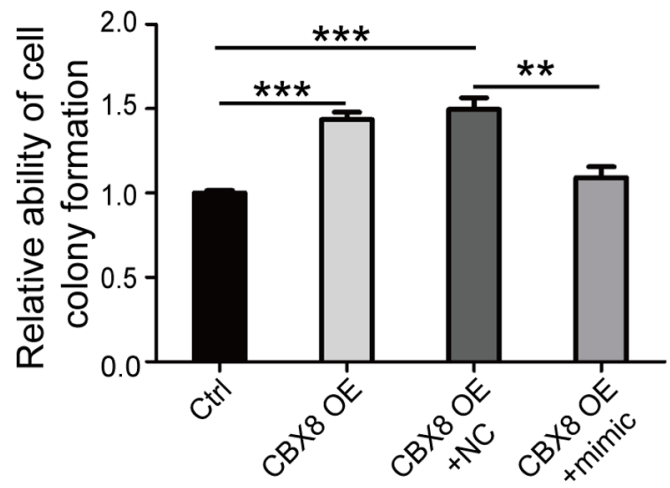

Figure 5. hsa-miR-429 antagonizes the positive effect of CBX8 on DLBCL cell proliferation and on the inhibition of apoptosis. (A) Western blot analysis revealed that hsa-miR-429 mimic significantly downregulated the protein expression levels of CBX8 in cells. (B) The transfection effect was detected by reverse transcription-quantitative PCR. (C) Cell Counting Kit-8 assays indicated that hsa-miR-429 mimic significantly inhibited the increase in proliferation of DLBCL cells following overexpression of CBX8. (D) Results of cell colony formation assay. (E) Recovery of the cell apoptotic rate by hsa-miR-429 mimic was detected by flow cytometry. (F) Recovery of the cell cycle distribution by hsa-miR-429 mimic was detected by flow cytometry. ${ }^{* *} \mathrm{P}<0.01$ and ${ }^{* * *} \mathrm{P}<0.001$. DLBCL, diffuse large B-cell lymphoma; hsa, Homo sapiens; miR, microRNA; CBX8, chromobox 8; UTR, untranslated region; OE, overexpression; Ctrl, control.

\section{Acknowledgements}

Not applicable.

\section{Funding}

No funding was received. 


\section{Availability of data and materials}

The datasets used and/or analyzed during the current study are available from the corresponding author on reasonable request.

\section{Authors' contributions}

YL and ZJY performed the experiments. ML, HML, JZZ, TX and YYT confirmed the authenticity of all the raw data. YL and ZJY performed the statistical analysis. ML, HML, JZZ, TX and YYT performed the bioinformatics analysis. ZPH designed the study and YL drafted the manuscript. All authors read and approved the final manuscript.

\section{Ethics approval and consent to participate}

The present study was approved by the Ethics Committee of Jingzhou Central Hospital (Jingzhou, China). All participants signed informed consent forms.

\section{Patient consent for publication}

Not applicable.

\section{Competing interests}

The authors declare that they have no competing interests.

\section{References}

1. Sehn LH and Gascoyne RD: Diffuse large B-cell lymphoma: Optimizing outcome in the context of clinical and biologic heterogeneity. Blood 125: 22-32, 2015.

2. Sehn LH and Salles G: Diffuse large B-cell lymphoma. N Engl J Med 384: 842-858, 2021

3. Maltby S, Plank M, Ptaschinski C, Mattes J and Foster PS: MicroRNA function in mast cell biology: Protocols to characterize and modulate microRNA expression. Methods Mol Biol 1220: 287-304, 2015 .

4. Hata A and Lieberman J: Dysregulation of microRNA biogenesis and gene silencing in cancer. Sci Signal 8: re3, 2015.

5. Cheng CJ, Bahal R, Babar IA, Pincus Z, Barrera F, Liu C, Svoronos A, Braddock DT, Glazer PM, Engelman DM, et al: MicroRNA silencing for cancer therapy targeted to the tumour microenvironment. Nature 518: 107-110, 2015.

6. Xue H and Tian GY: miR-429 regulates the metastasis and EMT of HCC cells through targeting RAB23. Arch Biochem Biophys 637: 48-55, 2018.

7. Guo C, Zhao D, Zhang Q, Liu S and Sun MZ: miR-429 suppresses tumor migration and invasion by targeting CRKL in hepatocellular carcinoma via inhibiting Raf/MEK/ERK pathway and epithelial-mesenchymal transition. Sci Rep 8: 2375, 2018.

8. Zhang C, Chang C, Gao H, Wang Q, Zhang F and Xu C: miR-429 regulates rat liver regeneration and hepatocyte proliferation by targeting JUN/MYC/BCL2/CCND1 signaling pathway. Cell Signal 50: 80-89, 2018

9. Zhang M, Dong BB, Lu M, Zheng MJ, Chen H, Ding JZ, $\mathrm{Xu} \mathrm{AM}$ and $\mathrm{Xu} \mathrm{YH}$ : miR-429 functions as a tumor suppressor by targeting FSCN1 in gastric cancer cells. OncoTargets Ther 9: 1123-1133, 2016

10. Wu CL, Ho JY, Hung SH and Yu DS: miR-429 expression in bladder cancer and its correlation with tumor behavior and clinical outcome. Kaohsiung J Med Sci 34: 335-340, 2018.
11. Yang J, Liu Y, He A, Liu Y, Wu J, Liao X, Lv Z, Wang F and Mei H: hsa-miR-429 promotes bladder cancer cell proliferation via inhibiting CDKN2B. Oncotarget 8: 68721-68729, 2017.

12. Deng Y, Luan F, Zeng L, Zhang Y and Ma K: miR-429 suppresses the progression and metastasis of osteosarcoma by targeting ZEB1. EXCLI J 16: 618-627, 2017.

13. Wang HF, Wang WH, Zhuang HW and Xu M: miR-429 regulates the proliferation and apoptosis of nephroblastoma cells through targeting c-myc. Eur Rev Med Pharmacol Sci 22: 5172-5179, 2018.

14. Liu D, Song L, Dai Z, Guan H, Kang H, Zhang Y, Yan W, Zhao X and Zhang S: miR-429 suppresses neurotrophin-3 to alleviate perineural invasion of pancreatic cancer. Biochem Biophys Res Commun 505: 1077-1083, 2018.

15. Zong M, Liu Y, Zhang K, J Y and Chen L: The effects of miR-429 on cell migration and invasion by targeting Slug in esophageal squamous cell carcinoma. Pathol Res Pract 215: 152526, 2019.

16. Wang Y, Liu J, Yao Q, Wang Y, Liu Z and Zhang L: LncRNA SNHG6 promotes Wilms' tumor progression through regulating miR-429/ FRS2 axis. Cancer Biother Radiopharm: Jan 22, 2021 (Epub ahead of print).

17. Zhang L, Liu Q, Mu Q, Zhou D, Li H, Zhang B and Yin C: miR-429 suppresses proliferation and invasion of breast cancer via inhibiting the $\mathrm{Wnt} / \beta$-catenin signaling pathway. Thorac Cancer 11: 3126-3138, 2020.

18. Cava C, Novello C, Martelli C, Lodico A, Ottobrini L, Piccotti F, Truffi M, Corsi F, Bertoli G and Castiglioni I: Theranostic application of miR-429 in HER $^{2+}$ breast cancer. Theranostics 10: 50-61, 2020.

19. Livak KJ and Schmittgen TD: Analysis of relative gene expression data using real-time quantitative PCR and the 2(-Delta Delta C(T)) Method. Methods 25: 402-408, 2001.

20. Zhang CZ, Chen SL, Wang CH, He YF, Yang X, Xie D and Yun JP: CBX8 exhibits oncogenic activity via AKT/ $\beta$-catenin activation in hepatocellular carcinoma. Cancer Res 78: 51-63, 2018.

21. Tang B, Tian Y, Liao Y, Li Z, Yu S, Su H, Zhong F, Yuan G, Wang Y, Yu H, et al: CBX8 exhibits oncogenic properties and serves as a prognostic factor in hepatocellular carcinoma. Cell Death Dis 10: 52, 2019.

22. Xiao L, Zhou Z, Li W, Peng J, Sun Q, Zhu H, Song Y, Hou JL, Sun J, Cao HC, et al: Chromobox homolog 8 (CBX8) Interacts with Y-Box binding protein 1 (YBX1) to promote cellular proliferation in hepatocellular carcinoma cells. Aging (Albany NY) 11: 7123-7149, 2019.

23. Zhang $\mathrm{Y}$, Chen $\mathrm{H}$, Zhu $\mathrm{H}$ and Sun $\mathrm{X}$ : CBX8 promotes tumorigenesis and confers radioresistance in esophageal squamous cell carcinoma cells through targeting APAF1. Gene 711: 143949, 2019

24. Xiao W, Ou C, Qin J, Xing F, Sun Y, Li Z and Qiu J: CBX8, a novel DNA repair protein, promotes tumorigenesis in human esophageal carcinoma. Int J Clin Exp Pathol 7: 4817-4826, 2014.

25. Tang J, Wang G, Zhang M, Li FY, Sang Y, Wang B, Hu K, Wu Y, Luo R, Liao D, et al: Paradoxical role of CBX8 in proliferation and metastasis of colorectal cancer. Oncotarget 5: 10778-10790, 2014.

26. Lee SH, Um SJ and Kim EJ: CBX8 suppresses Sirtinol-induced premature senescence in human breast cancer cells via cooperation with SIRT1. Cancer Lett 335: 397-403, 2013.

27. Lee SH, Um SJ and Kim EJ: CBX8 antagonizes the effect of Sirtinol on premature senescence through the AKT-RB-E2F1 pathway in K562 leukemia cells. Biochem Biophys Res Commun 469: 884-890, 2016.

28. Zeng F, Luo L, Li D, Guo J and Guo M: KPNA2 interaction with CBX8 contributes to the development and progression of bladder cancer by mediating the PRDM1/c-FOS pathway. J Transl Med 19: 112, 2021

29. Wang S, Tailor K and Kwabi-Addo B: Androgen-induced epigenetic profiles of polycomb and trithorax genes in prostate cancer cells. Anticancer Res 40: 2559-2565, 2020.

(i) (8) This work is licensed under a Creative Commons c. International (CC BY-NC-ND 4.0) License. 